\title{
A discrepancy measure for segmentation evaluation from the perspective of object recognition
}

\author{
Jian Yang, Yuhong He, John Caspersen, Trevor Jones \\ Version Post-print/Accepted Manuscript \\ Citation Jian Yang, Yuhong He, John Caspersen, Trevor Jones. A discrepancy \\ (published version) measure for segmentation evaluation from the perspective of object \\ recognition. ISPRS Journal of Photogrammetry and Remote Sensing. \\ Volume 101, 2015. doi: https://doi.org/10.1016/i.isprsiprs.2014.12.015 \\ DOI: https://doi.org/10.1016/j.isprsjprs.2014.12.015

\begin{abstract}
Copyright/License

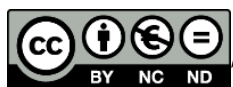
Attribution-NonCommercial-NoDerivatives 4.0 International License. To view a copy of this license, visit Creative Commons BY NC ND 4.0 License.
\end{abstract}

\section{How to cite TSpace items}

Always cite the published version, so the author(s) will receive recognition through services that track citation counts, e.g. Scopus. If you need to cite the page number of the author manuscript from TSpace because you cannot access the published version, then cite the TSpace version in addition to the published version using the permanent URI (handle) found on the record page.

This article was made openly accessible by $U$ of $T$ Faculty. Please tell us how this access benefits you. Your story matters. 
1

2

3

4

$5{ }^{1}$ Department of Geography, University of Toronto, 100 St. George Street, Toronto, ON M5S 3G3,

6 Canada.

$7 \quad{ }^{2}$ Department of Geography, University of Toronto Mississauga, 3359 Mississauga Rd North,

8 Mississauga, ON L5L 1C6, Canada.

$9{ }^{3}$ Faculty of Forestry, University of Toronto, 33 Willcocks Street, Toronto, ON M5S 3B3, Canada.

10

11

12

13

14

15

16

17

18

19

20

21

22

23
Jian Yang ${ }^{1,2, *}$, Yuhong He ${ }^{2}$, John Caspersen ${ }^{3}$, Trevor Jones ${ }^{4}$

${ }^{4}$ Ontario Forest Research Institute, Ministry of Natural Resources, 1235 Queen Street East, Sault Ste

Marie, ON, P6A 2E5, Canada.

* Corresponding author. Tel.: +1 4169783375.

Email address: jiangeo.yang@mail.utoronto.ca (J. Yang). 6 7 8

9

21

\section{2} 3 


\section{Abstract}

Image segmentation is one of the most important components within the framework of object

26 based image analysis. Segmentation evaluation thus plays a critical role in controlling the quality of object

27 based image analysis workflow. Among a variety of segmentation evaluation methods and criteria,

28 discrepancy measurement is believed to be the most useful and commonly employed in many applications.

29 However, existing measures have largely ignored the importance of object recognition in segmentation

30 evaluation. In this study, a new discrepancy measure of Segmentation Evaluation Index (SEI) is proposed

31 to overcome this limitation by redefining the corresponding segment using the condition of two-side $50 \%$

32 overlap instead of one-side $50 \%$ overlap that has been commonly used. The effectiveness of SEI is further

33 investigated using the schematic segmentation cases and remote sensing images. Results demonstrate that,

34 the proposed SEI outperforms the other two existing discrepancy measures in terms of object recognition 35 accuracy and identification of detailed segmentation difference.

36

37 Keywords: image segmentation, quantitative evaluation, discrepancy measure, object recognition, 38 corresponding segment, Segmentation Evaluation Index (SEI) 


\section{Introduction}

As is widely accepted, image segmentation is the first, fundamental, and critical step for object based image analysis. It is a process of partitioning the entire image into a number of non-overlapping segments for the subsequent object recognition, image classification, or information extraction. In the past decades, a variety of popular segmentation algorithms have been proposed, such as watershed segmentation (Li et al., 2010; Li \& Xiao, 2007; Vincent \& Soille, 1991), mean-shift segmentation (Comaniciu \& Meer, 2002), and the fractal net evolution approach (Baatz \& Schäpe, 2000; Benz et al., 2004). Nevertheless, it still remains challenging and problematic to integrate a comprehensive framework for segmentation evaluation (Ryherd \& Woodcock, 1996; Shandley, Franklin \& White, 1996).

In general, the methods of segmentation evaluation include visual inspection, quantitative evaluation, and indirect evaluation (e.g., classification accuracy) (Li et al., 2011). Because human eyes are considered as a strong and experienced source, visual inspection is a commonly-used evaluation method although it might be subjective and qualitative (Pesaresi \& Benediktsson, 2001; Zhang, Fritts \& Goldman, 2008). On the other hand, classification accuracy can imply the quality of image segmentation in some way, but it is not a direct indication of the segmentation accuracy (Kim, Madden \& Warner, 2009; Zhang et al., 2005). Despite some popularity of visual inspection and indirect evaluation, quantitative evaluation has attracted growing attention for segmentation evaluation through providing more detailed information (e.g., over-segmentation and under-segmentation).

Quantitative evaluation methods can be categorized into three types: analytical, empirical goodness, and empirical discrepancy, among which empirical discrepancy has been proven the most effective and widely used (Zhang, 1996). To assess image segmentation, the evaluation based on empirical discrepancy measures the dissimilarity between a reference polygon and a corresponding segment. A few discrepancy measures were first proposed to quantify their overlapping area (Carleer, Debeir \& Wolff, 
2005; Clinton et al., 2010; Lucieer \& Stein, 2002; Möller, Lymburner \& Volk, 2007; Weidner, 2008; Zhan et al., 2005). These overlapping area based measures, later categorized as geometric discrepancy (Liu et al., 2012), are able to characterise the non-overlapping area of a reference polygon and a corresponding segment (Fig. 1a), however, they require that a reference polygon should only have one corresponding segment. In reality, it is very common for a reference polygon to have a number of corresponding segments (Fig. 1b). The prerequisite of one reference to only one corresponding segment is thus almost impossible to satisfy when using the geometric discrepancy measures. The flaw in the geometric discrepancy measures has been recently addressed using newly proposed indices which consider arithmetic discrepancy ( Liu et al., 2012; \& Yang, Li and He, 2014). Arithmetic discrepancy is defined as the number of corresponding segments for any reference polygon (Liu et al., 2012).

In general, the discrepancy measures that are proposed by previous studies label a candidate segment as the corresponding segment of a reference polygon when the overlapping area is over $50 \%$ of EITHER the reference polygon OR the candidate segment (thereafter defined as one-side 50\% overlap) (Clinton et al., 2010; Liu et al., 2012; Yang, Li \& He, 2014). However, the use of one-side 50\% overlap in the discrepancy measures would not work in many segmentation cases, due to the ignorance of object recognition in segmentation evaluation. Using two segmentation results for a polygon as an example (Fig. $1 \mathrm{~b}$ and 1c), all segments (in blue) in the two cases can be considered as corresponding segments for the reference polygon (in red) because the overlapping area is over 50\% (in fact 100\%) of each candidate segment and the one-side $50 \%$ overlap prerequisite for the discrepancy measures is thus satisfied. Consequently, the two segmentation results for the polygon are exactly the same in terms of geometric discrepancy (i.e. the non-overlapping areas are the same) and arithmetic discrepancy (i.e. three segments corresponding to one reference polygon are the same in both cases). However, it is obvious that the candidate segments in Fig. 1c contribute a higher segmentation quality than those in Fig. 1b because the 
93 largest segment in Fig. 1c can better recognize the reference polygon. This example clearly demonstrated 94 that the current discrepancy measures cannot efficiently recognize objects as a result of only utilizing one95 side $50 \%$ overlap. To differentiate these two cases, a new discrepancy (thereafter defined as object96 recognized discrepancy) is needed to identify whether or not a corresponding segment is able to well 97 recognize a reference object. Differing from geometric and arithmetic discrepancy, the new discrepancy 98 will incorporate the object recognition information for a correctly recognized object through the prerequisite that the overlapping area between a reference polygon and the candidate segment has to be more than $50 \%$ of BOTH the reference polygon AND the candidate segment (Lamar, McGraw \& Warner, $1012005)$.

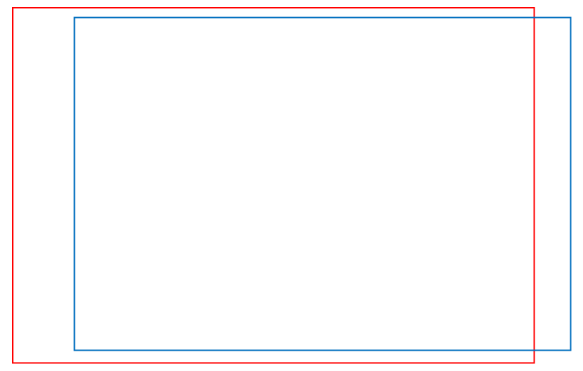

(a)

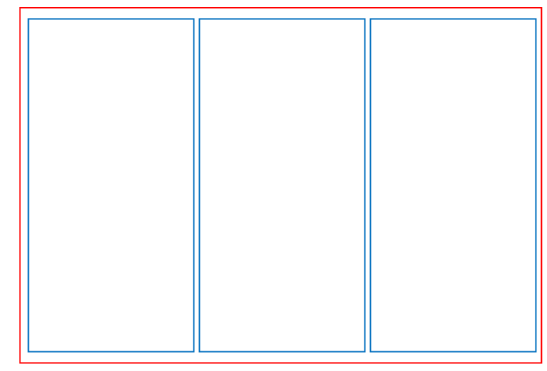

(b)

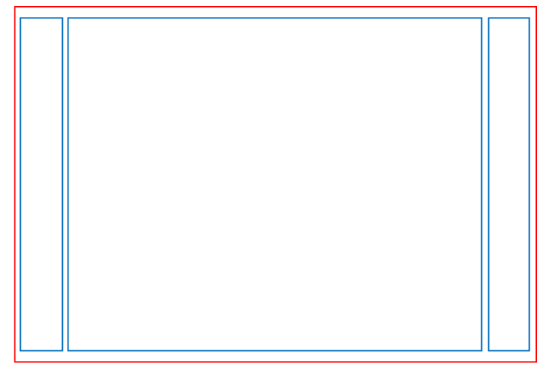

(c)
102

103

104 105 106 107 108 109 110

As far as we know, no study has yet incorporated the information of object recognition into the discrepancy measures, in spite of the fact that object recognition is a most important criterion when measuring the discrepancy between a reference object and a corresponding segment. It is thus of great necessity to identify if the reference object is correctly recognition before a detailed discrepancy measurement is calculated. In order to address this knowledge gap, we propose a new discrepancy measure 
111 to evaluate the quality of image segmentation which takes into account geometric discrepancy, arithmetic

112 discrepancy, and object-recognized discrepancy. Schematic segmentation cases and remote sensing 113 images are used to examine the performance of the proposed index in comparison with the other two 114 existing discrepancy measures (Liu et al., 2012; Yang, Li \& He, 2014).

\section{Discrepancy measures}

Since object recognition is one of the most important objectives of image segmentation, this study adopted the two-side $50 \%$ overlap as the prerequisite to identify the corresponding segment for a reference polygon. Under this context, the reference polygon without any corresponding segment will be considered as an omitted or missing object in the process of segmentation evaluation. and Euclidian Distance 2 (ED2) (Liu et al., 2012).

$$
\begin{aligned}
& P S E=\frac{\operatorname{area}(S-R)}{\operatorname{area}(R)} \\
& N S R=\frac{|m-v|}{m} \\
& E D 2=\sqrt{P S E^{2}+N S R^{2}}
\end{aligned}
$$

where $R$ and $S$ are the datasets of reference polygons and corresponding segments while $m$ and $v$ are the numbers of reference polygons and corresponding segments. A PSE value of zero implies that there is no under-segment, and a NSR value of zero means an optimal one-to-one relationship between the reference polygons and corresponding segments.

Although ED2 considers both geometric and arithmetic matches, the difficulty of PSE and NSR normalization can result in the exaggeration of over-segmentation at finer scales when NSR overwhelms PSE (Yang, Li \& He, 2014). In addition, the compensation effect caused by the coexistence of one-to- 
many over-segmentation and many-to-one under-segmentation can result in invalid NSR. For instance,

133 since the one-side $50 \%$ overlap fails to ensure that there is at least a candidate segment corresponding to 134 any one reference polygon, the over-segmentation of other reference objects can compensate this effect 135 on the NSR even if there is an omitted or missing reference object. Thus, Yang, Li and He (2014) 136 developed the local metrics of OverSegmentation 2 (OS2), UnderSegmentation 2 (US2), and Euclidian 137 Distance $3($ ED3).

$$
\begin{aligned}
& O S 2=\overline{\sum_{i} \sum_{j}\left(1-\frac{\operatorname{area}\left(r_{i} \cap s_{j}\right)}{\operatorname{area}\left(r_{i}\right)}\right)}, s_{j} \in S \\
& U S 2=\overline{\sum_{i} \sum_{j}\left(1-\frac{\operatorname{area}\left(r_{i} \cap s_{j}\right)}{\operatorname{area}\left(s_{j}\right)}\right)}, s_{j} \in S \\
& E D 3=\sum_{i} \sum_{j} \sqrt{\frac{\left(1-\frac{\operatorname{area}\left(r_{i} \cap s_{j}\right)}{\operatorname{area}\left(r_{i}\right)}\right)^{2}+\left(1-\frac{\operatorname{area}\left(r_{i} \cap s_{j}\right)}{\operatorname{area}\left(s_{j}\right)}\right)^{2}}{2}}, s_{j} \in S
\end{aligned}
$$

139 where $r_{i}$ is an arbitrary element of $R$ and $s_{j}$ is the corresponding segment, as an element of $S$.

Despite the merits of ED2 and ED3 as discrepancy measures, both of them ignore the accuracy of 141 object recognition due to the use of one-side $50 \%$ overlap of corresponding segments. In this study, a new 142 measure of Segmentation Evaluation Index (SEI) was developed from ED3 using the corresponding 143 segments identified by the two-side $50 \%$ overlap.

$$
\begin{aligned}
& S E I_{\text {local }}(i)=\left\{\begin{array}{cl}
\sqrt{\frac{\left(1-\frac{\operatorname{area}\left(r_{i} \cap s_{i}\right)}{\operatorname{area}\left(r_{i}\right)}\right)^{2}+\left(1-\frac{\operatorname{area}\left(r_{i} \cap s_{i}\right)}{\operatorname{area}\left(s_{i}\right)}\right)^{2}}{2}}, & , s_{i} \in S_{2 \mathrm{~S}} \\
1 & , s_{i} \notin S_{2 \mathrm{~S}}
\end{array}\right. \\
& S E I=\frac{1}{n} \sum_{i=1}^{n} S E I_{\text {local }}(i)
\end{aligned}
$$


where $S E I_{\text {local }}(i)$ denotes the local $S E I$ for the $i t h$ reference polygon and $n$ denotes the number of reference polygons. $r_{i}$ is the ith reference polygon while $s_{i}$ is the corresponding segment, as an element of the two147 side $50 \%$ overlap of corresponding segment dataset $S_{2 S}$.

Under the prerequisite of two-side $50 \%$ overlap, it is not possible to identify more than one candidate segment corresponding to a given reference polygon. When a candidate segment correctly recognizes an object, $S E I_{\text {local }}$ is able to measure the discrepancy between the corresponding segment and 151 the reference polygon. A $S E I_{\text {local }}$ value of zero indicates perfect-segmentation, neither over-segmentation 152 nor under-segmentation. If there is no candidate segment corresponding to a given reference polygon, the 153 value of $S E I_{\text {local }}$ is defined as one, indicating that it is an omitted or missing object. Given the $S E I_{\text {local }}$ 154 values of all reference polygons, the value of SEI is ranging from zero to one. Apparently, a higher value 155 of SEI suggests an overall lower quality of image segmentation.

In this study, both schematic segmentation cases and remote sensing images were utilized to 157 examine the performance of SEI for discrepancy measurement, compared with the mentioned two metrics 158 of ED2 and ED3.

\section{Implementations}

\section{1: Schematic segmentation cases}

The proposed index (SEI) was first validated using five basic schematic cases (Fig. 2) including absolute over-segmentation, one-to-many over-segmentation, one-to-one perfect-segmentation, many-toone under-segmentation, and absolute under-segmentation. It is worth noting that the absolute oversegmentation represents the case that a reference polygon is divided into infinite segments while the absolute under-segmentation is for the case that infinite reference polygons are belong to a segment. When segmenting remote sensing images, the absolute over-segmentation does not exist because the smallest 
168 unit of an image is a pixel. Meanwhile, a finite number of objects within a remote sensing image make 169 the absolute under-segmentation impossible. Despite the inexistence of these two extreme cases in an 170 image, this study took both of them into consideration to enable a complete set of schematic segmentation 171 cases for validating the SEI.

172

(a)
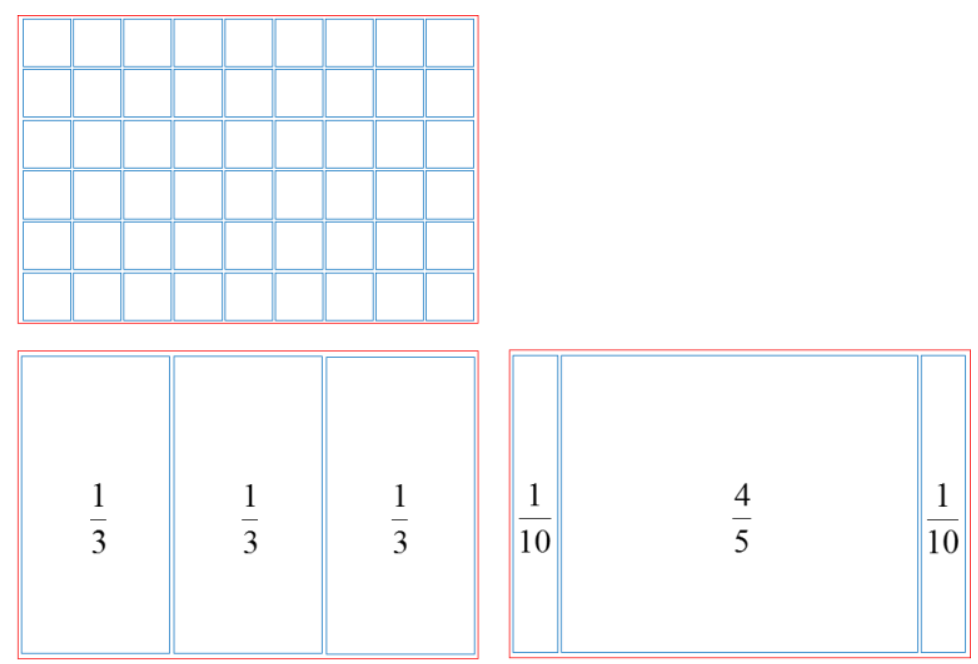

(c)
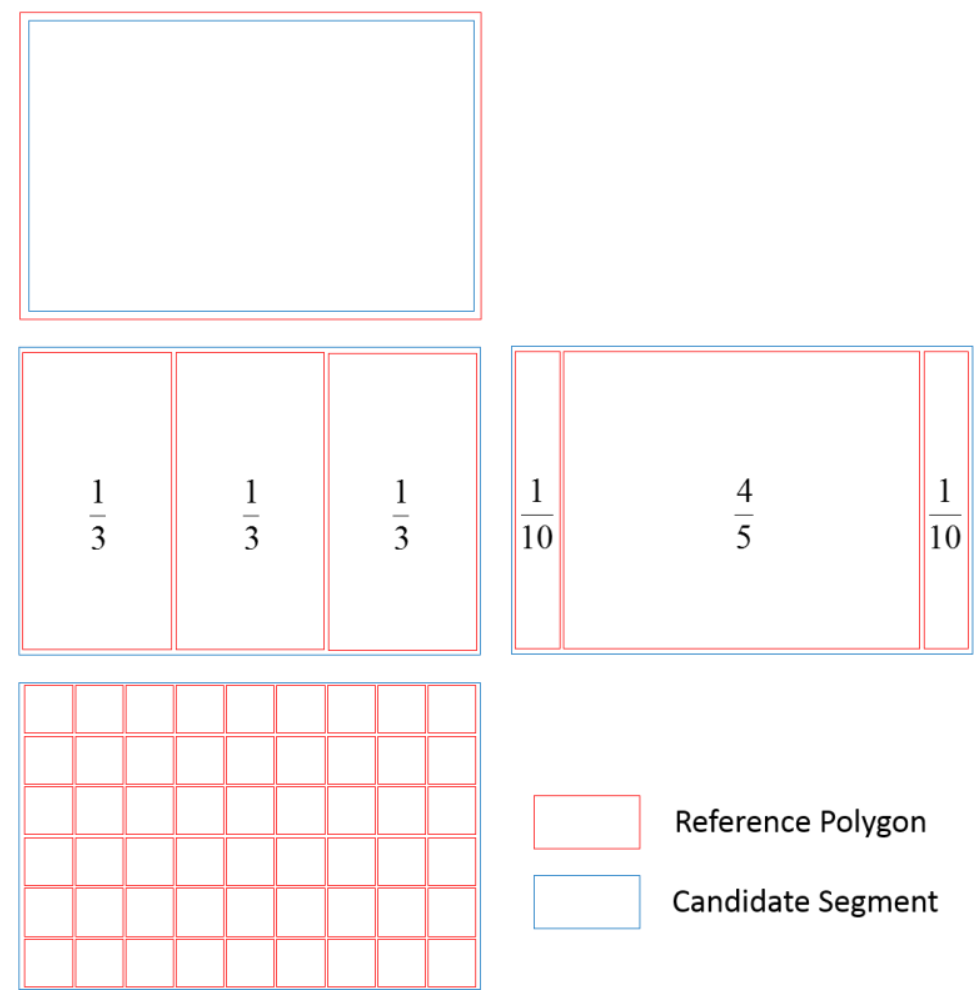

Reference Polygon

Candidate Segment 
174 Fig. 2. Schematic representation of the schematic segmentation cases: absolute over-segmentation (a), 175 one-to-many over-segmentation (b), one-to-one perfect-segmentation (c), many-to-one under176 segmentation (d), and absolute under-segmentation (e).

178 Table 1. Discrepancy measure calculation for absolute over-segmentation (AbOver), one-to-many over179 segmentation (Over), one-to-one perfect-segmentation (Perfect), many-to-one under-segmentation (Und), 180 and absolute under-segmentation (AbUnd).

\begin{tabular}{cccccccc}
\hline & AbOver & Over (left) & Over (right) & Perfect & Und (right) & Und (left) & AbUnd \\
\hline PSE & 0 & 0 & 0 & 0 & 0 & 0 & 0 \\
NSR & Infinite & 2 & 2 & 0 & 0.67 & 0.67 & 1 \\
ED2 & Infinite & 2 & 2 & 0 & 0.67 & 0.67 & 1 \\
OS2 & 1 & 0.47 & 0.47 & 0 & 0 & 0 & 0 \\
US2 & 0 & 0 & 0 & 0 & 0.47 & 0.47 & 1 \\
ED3 & 1 & 0.47 & 0.47 & 0 & 0.47 & 0.47 & 1 \\
SEI & 1 & 1 & 0.14 & 0 & 0.71 & 1 & 1 \\
\hline
\end{tabular}

The ED2, ED3, and SEI for the schematic segmentation cases are calculated and listed in Table 1.

183 All the discrepancy measures $(E D 2, E D 3$, and $S E I)$ are successful in characterizing the three extreme cases 184 including absolute over-segmentation, one to one perfect segmentation, and absolute under-segmentation, 185 although the ranges of values are not the same (Table 1). For instance, the value of ED2 is infinite for the 186 absolute over-segmentation representation whereas both ED3 and SEI are one. Further, the value of ED2 187 is not completely symmetrical: ranging from zero to infinite in the over-segmentation case while from 188 zero to one in the under-segmentation case. This result indicates that both SEI and ED3 can characterize 
the three extreme cases perfectly, while $E D 2$ is too sensitive for over-segmentation at finer scales but less sensitive for under-segmentation at coarser scales, which is also consistent with the results in Witharana and Civco (2014). In other words, it is very likely that ED2 amplifies the effectiveness of oversegmentation in contrast to that of under-segmentation (Yang, Li \& He, 2014).

The proposed SEI is able to show advantages over the other two measures when evaluating the segmentation in more common-seen cases (i.e. one-to-many over-segmentation and many-to-one undersegmentation), demonstrated by below examples. The over-segmentation example in Fig. $2 \mathrm{~b}$ where a reference polygon (in Red) is divided into three segments (in Blue) in two segmentation cases (left and right), but the segments vary in area proportion between the cases. The right segmentation case is apparently better than the left one from the perspective of object recognition, because the largest segment that occupies $4 / 5$ of the reference polygon in the right case is able to represent it, whereas none of segments on the left recognizes the reference polygon. However, neither ED2 nor ED3 is able to identify the difference because they conclude the same value for both cases (a value of 2 for ED2 and 0.47 for ED3, Table 1). That is because the one-side 50\% overlap allows all the three segments in these two cases becoming the corresponding segments when calculating ED2 and ED3. On the contrary, the proposed SEI, which uses the two-side 50\% overlap as the prerequisite, overcomes the limitation of $E D 2$ and $E D 3$, and produced a value of 1 (an indication of the worse quality) for the left segmentation case and a value of 0.14 (an indication of the better quality) for the right segmentation case. In the under-segmentation cases (Fig. 2d left and right), only the $S E I$ is sensitive enough to detect the dissimilarity of two undersegmentation cases. The ED2 and ED3 show no differences in their values between two cases (i.e. 0.67 for ED2 and 0.47 for ED3 in both cases). Differently, the SEI (the value of 1) for the left undersegmentation case is higher than that (0.71) for the right under-segmentation cases. The difference of SEI values is mainly attributed from object recognition. 
In addition, the $S E I$ value of 0.14 in the over-segmentation case (Fig. 2b right) is lower than that

213 of 0.71 in the under-segmentation case (Fig. 2d right). This is expected because one object out of one is 214 recognized in the over-segmentation case (Fig. 2b right) while only one object out of three is recognized 215 in the under-segmentation case (Fig. 2d right). It is thus reasonable that the former one represents a higher 216 quality of image segmentation. investigation of schematic segmentation cases. Further examination is conducted using the remote sensing images from both urban and natural forested areas.

\subsection{Experiment 2: remote sensing images}

3.2.1 Image data preprocessing

Two image datasets were adopted to examine the effectiveness of the proposed SEI in 
235 infrared. In order to investigate the performance of $S E I$, two subsets of $1000 \times 1000$ pixels were clipped 236 from the pan-sharpened WorldView-2 satellite (Fig. 3a) and ADS40 aerial (Fig. 3b) images, respectively.

237 Particularly, the urban scene describes a typical built-up area with trees, grass and many high-rise 238 buildings, and the forest scene characterizes a deciduous stand with different types of broad-leaved trees.

239 The diversity of land cover objects makes it suitable to verify the robustness and transferability of the 240 proposed $S E I$ in segmentation evaluation.

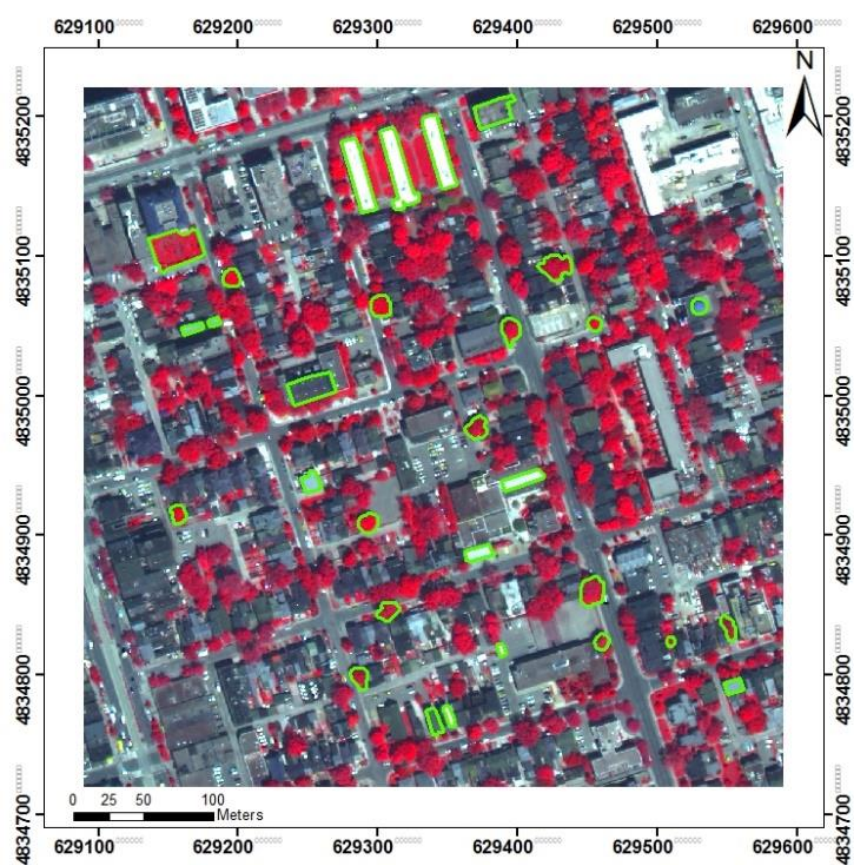

(a)

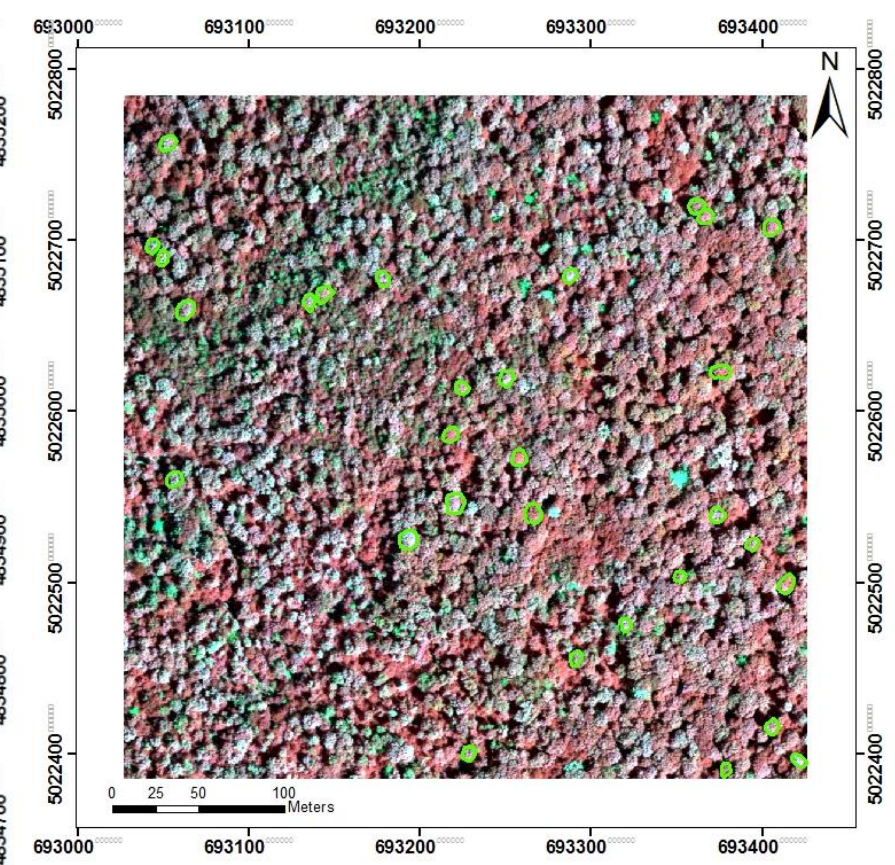

(b)

243 Fig. 3. Pan-sharpened WorldView-2 satellite image of downtown Toronto with a band combination of

244 near infrared 1, red, and green as R, G, and B (a), and ADS40 aerial image of Haliburton Forest with a 245 band combination of near infrared, red, and green as R, G, and B (b). Both images are under WGS84 UTM 246 coordinate system. Green polygons represent the manually digitized reference objects. 
This study employed the BerkeleyImageSeg software package (BIS; http://www.imageseg.com)

to produce the appropriate segmentation for the above two remote sensing images. The program utilized the region merging algorithm proposed by Benz et al. (2004) to partition the input image into segments based on three parameters of threshold, shape (0.1-0.9), and compactness (0.1-0.9). While a higher threshold indicates the larger size of segments, the higher shape and compactness result in the more round and smooth segments, respectively. After a series of trial-and-error parameter selection, we chose to use $100,0.5$, and 0.5 as threshold, shape, and compactness for the segmentation of pan-sharpened WorldView2 satellite image, and 15, 0.9, and 0.9 as threshold, shape, and compactness for the segmentation of ADS40 aerial image. In comparison with the urban scene, the forest scene required a finer threshold due to the smaller size and less distinct boundary of tree crowns. Moreover, the highest shape and compactness ensured the good roundness and smoothness of tree crowns in the forest scene.

\subsubsection{Segmentation evaluation}

For segmentation evaluation, a total of 30 reference polygons was randomly delineated for each scene (Fig. 3). Particularly, the reference objects in the urban scene include vegetation and impervious rooftops while the reference objects in the forest scene consist of individual deciduous tree crowns. As mentioned above, the proposed SEI considers two-side $50 \%$ overlaps while ED2 and ED3 only consider one-side $50 \%$ overlap as the condition to identify corresponding segments. In order to observe the performance of SEI for discrepancy measurement, this study adjusted the overlapping area from $51 \%$ to 91\% with an interval of 5\% and calculated all three measures (the SEI, ED2 and ED3 in Fig. 4). Since a larger overlapping area indicates a higher criterion of segmentation evaluation, fewer candidate segments can become the corresponding segments of a given set of reference polygons. Theoretically, an ideal discrepancy measure should be able to capture this tendency, the quality of image segmentation substantially decreasing as the overlapping area raises. 
The results in Fig. 4 indicated that only SEI is able to capture the decreased quality of image 273 segmentation as the overlapping area raises in both urban and forest scenes. In the urban scene (Fig. 4a), 274 the decreasing tendency of $E D 2$ or $E D 3$ implies an increasing quality of image segmentation, which is not 275 correct but expected because both measures would detect lower number but higher quality of 276 corresponding segments as the overlapping areas increases, at an expense of ignoring reference polygons 277 without any corresponding segments. Addressing the issue in the ED2 and ED3, the proposed SEI uses 278 one to represent an omitted or missing object (i.e. no corresponding segments) and thus accurately 279 characterizes the tendency with the increasing value from 0.49 to 0.91 . In the forest scene (Fig. $4 \mathrm{~b}$ ), ED2 280 or ED3 failed to identify the variation of segmentation quality. A V-shape reversal of ED2 value emerges 281 around the overlapping area of $76 \%$. At that point, the number of corresponding segments determined by 282 the one-side overlap is just equal to the number of reference polygons. So the NSR value of zero makes 283 the ED2 value the least there.
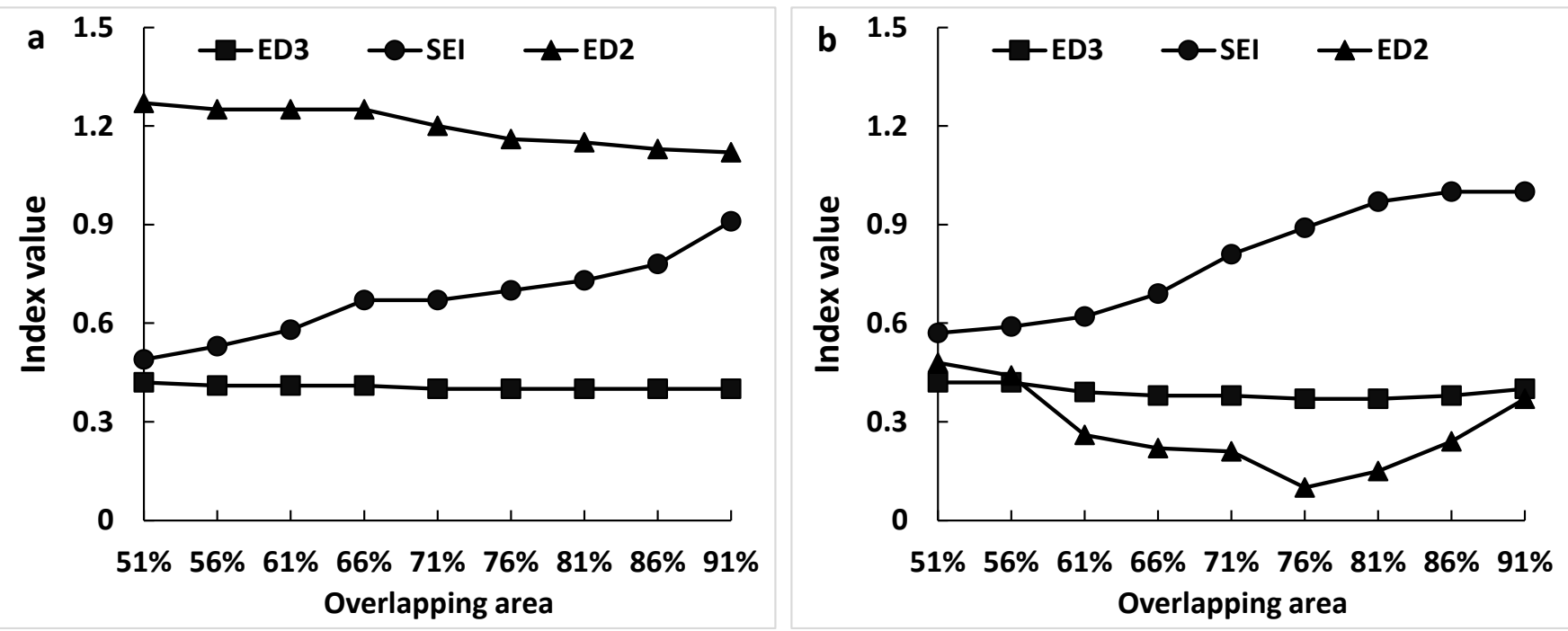

Fig. 4. Discrepancy measures versus the increasing overlapping area in both urban (a) and forest scenes 287 (b). 
In summary, the proposed SEI not only takes the object recognition into consideration as indicated 289 in the schematic cases, but also provides valid discrepancy measurement which is not possible through 290 the utilization of the other two indices.

\section{Conclusions}

When evaluating the quality of image segmentation, it is of great significance to measure the 294 dissimilarity between a reference polygon and a corresponding segment using the appropriate metric. 295 However, a higher priority should be assigned to object recognition by determining whether a reference object is correctly recognized before discrepancy measurement. In this study, a new index of SEI was the performance of $S E I$. The results showed that the proposed SEI was more effective to consider object recognition accuracy and to identify detailed segmentation difference, in comparison with the existing discrepancy measures of ED2 and ED3, that it can be commonly applied to evaluate image segmentation for different types of landscapes based on the verification of schematic segmentation cases. Additionally, the discrepancy measure of SEI is also 305 promising as a supervised method for selecting the optimal segmentation scale in object based image 306 analysis, which will be further implemented in the future work.

\section{Acknowledgements}

Support for this study was provided by NSERC Discovery Grant to Dr. Yuhong He in the 309 University of Toronto, the Ontario Forest Research Institute (OFRI), and the Ministry of Natural Resources (MNR), Canada. 


\section{References}

312 Baatz, M., \& Schäpe, A. (2000). Multiresolution segmentation: an optimization approach for high quality multi-scale image segmentation. Angewandte Geographische Informationsverarbeitung XII, 12-23.

Benz, U.C., Hofmann, P., Willhauck, G., Lingenfelder, I., \& Heynen, M. (2004). Multi-resolution, object-oriented fuzzy analysis of remote sensing data for GIS-ready information. ISPRS Journal of Photogrammetry and Remote Sensing, 58, 239-258.

Brower, B.V., \& Laben, C.A. (2000). Process for enhancing the spatial resolution of multispectral imagery using pan-sharpening. U.S. Patent 6011 875, Jan. 4, 2000.

Carleer, A., Debeir, O., \& Wolff, E. (2005). Assessment of very high spatial resolution satellite image segmentations. Photogrammetric Engineering and Remote Sensing, 71, 1285-1294.

Clinton, N., Holt, A., Scarborough, J., Yan, L., \& Gong, P. (2010). Accuracy assessment measures for object-based image segmentation goodness. Photogrammetric Engineering and Remote Sensing, 76, 289-299.

Comaniciu, D., \& Meer, P. (2002). Mean shift: A robust approach toward feature space analysis. IEEE Transactions on Pattern Analysis and Machine Intelligence, 24, 603-619.

Kim, M., Madden, M., \& Warner, T.A. (2009). Forest type mapping using object-specific texture measures from multispectral IKONOS imagery: Segmentation quality and image classification issues. Photogrammetric Engineering and Remote Sensing, 75, 819-829.

Lamar, W.R., McGraw, J.B., \& Warner, T.A. (2005). Multitemporal censusing of a population of eastern hemlock $(<\mathrm{i}>$ Tsuga canadensis $</ \mathrm{i}>\mathrm{L}$.) from remotely sensed imagery using an automated segmentation and reconciliation procedure. Remote Sensing of Environment, 94, 133143. 
334 Li, D., Zhang, G., Wu, Z., \& Yi, L. (2010). An edge embedded marker-based watershed algorithm for high spatial resolution remote sensing image segmentation. IEEE Transactions on Image Processing, 19, 2781-2787.

Li, P., Guo, J., Song, B., \& Xiao, X. (2011). A multilevel hierarchical image segmentation method for urban impervious surface mapping using very high resolution imagery. IEEE Journal of Selected Topics in Applied Earth Observations and Remote Sensing, 4, 103-116.

Li, P., \& Xiao, X. (2007). Multispectral image segmentation by a multichannel watershed - based approach. International Journal of Remote Sensing, 28, 4429-4452.

Liu, Y., Bian, L., Meng, Y., Wang, H., Zhang, S., Yang, Y., Shao, X., \& Wang, B. (2012). Discrepancy measures for selecting optimal combination of parameter values in object-based image analysis. ISPRS Journal of Photogrammetry and Remote Sensing, 68, 144-156.

Lucieer, A., \& Stein, A. (2002). Existential uncertainty of spatial objects segmented from satellite sensor imagery. IEEE Transactions on Geoscience and Remote Sensing, 40, 2518-2521.

Möller, M., Lymburner, L., \& Volk, M. (2007). The comparison index: A tool for assessing the accuracy of image segmentation. International Journal of Applied Earth Observation and Geoinformation, 9, 311-321.

Pesaresi, M., \& Benediktsson, J.A. (2001). A new approach for the morphological segmentation of highresolution satellite imagery. IEEE Transactions on Geoscience and Remote Sensing, 39, 309320.

Ryherd, S., \& Woodcock, C. (1996). Combining spectral and texture data in the segmentation of remotely sensed images. Photogrammetric Engineering and Remote Sensing, 62, 181-194. 
Shandley, J., Franklin, J., \& White, T. (1996). Testing the Woodcock-Harward image segmentation algorithm in an area of southern California chaparral and woodland vegetation. International Journal of Remote Sensing, 17, 983-1004.

Vincent, L., \& Soille, P. (1991). Watersheds in digital spaces: an efficient algorithm based on immersion simulations. IEEE Transactions on Pattern Analysis and Machine Intelligence, 13, 583-598.

Weidner, U. (2008). Contribution to the assessment of segmentation quality for remote sensing applications. International Archives of Photogrammetry, Remote Sensing and Spatial Information Sciences, 37, 479-484.

Witharana, C., \& Civco, D.L. (2014). Optimizing multi-resolution segmentation scale using empirical methods: Exploring the sensitivity of the supervised discrepancy measure Euclidean distance 2 (ED2). ISPRS Journal of Photogrammetry and Remote Sensing, 87, 108-121.

Yang, J., Li, P., \& He, Y. (2014). A multi-band approach to unsupervised scale parameter selection for multi-scale image segmentation. ISPRS Journal of Photogrammetry and Remote Sensing, Accepted.

Zhan, Q., Molenaar, M., Tempfli, K., \& Shi, W. (2005). Quality assessment for geo - spatial objects derived from remotely sensed data. International Journal of Remote Sensing, 26, 2953-2974.

Zhang, H., Fritts, J.E., \& Goldman, S.A. (2008). Image segmentation evaluation: A survey of unsupervised methods. Computer Vision and Image Understanding, 110, 260-280.

Zhang, Q., Pavlic, G., Chen, W., Fraser, R., Leblanc, S., \& Cihlar, J. (2005). A semi-automatic segmentation procedure for feature extraction in remotely sensed imagery. Computers \& Geosciences, 31, 289-296.

Zhang, Y.J. (1996). A survey on evaluation methods for image segmentation. Pattern Recognition, 29, $1335-1346$. 
